\title{
THE EFFECTS OF THE ROTATION IN PLASMA
}

\author{
S. N. Arteha \\ Space Research Institute, Profsoyuznaya 84/ 32, Moscow 117810, Russia
}

\begin{abstract}
The electric and magnetic self-fields can exist in the rotating plasma. The self-sustained rotation can be established in the plasma. The disturbed distribution function of rotating plasma is derived from the Vlasov equation. The propagation of waves in rotating plasma differs from that in the usual plasma. New terms of the Landau damping appear. The local rotational behaviour may become prevailing.
\end{abstract}

\section{INTRODUCTION}

Phenomena dealt with are not too rare. However, the causes of the phenomena (forces, fields) are rather weak and can often be supposed to be negligible.

It is well known that many astronomical and laboratory objects represent rotating plasma. Therefore, the study of rotating plasma is of great theoretical and practical interest.

It is assumed that plasma is neutral on the average. However, electric and magnetic fields can exist in plasma, because there are forces which oppose the electric one $\mathrm{e}^{1,2}$.

It is well known that in a wide variety of plasma devices plasma travels across the magnetic field at rates much higher than those explained by collisional processes. It has been assumed that the electron transport across the magnetic field arises from the $\vec{E} \times \vec{B}$ drift $\left(\vec{E} \times \vec{B}\right.$ instability $\left.^{3}\right)$.

The radial electric field exists to balance out the $\vec{v} \times \vec{B}$ and centrifugal force terms ${ }^{4}$.

The plane sheet stability of a surface charge has been considered for externally applied and mutually perpendicular fields $(\vec{E} \perp \vec{B})$ which produce an equilibrium particle flow in the sheet ${ }^{5}$. The calculation was extended to a cylindrical shell in the inhomogeneous magnetic field ${ }^{6}$ (the size of a shell is identical to the particle gyro-orbit).

The equilibrium properties induce a self-electric field for a charge slab or a cylinder (the fluid approximation ${ }^{7,8}$ ).

The effect of equilibrium radial electric field on the trapped - particle stability has been investigated ${ }^{9}$. The stabilization can result from the configuration 
in which the radial potential assumes a hollow profile (the equilibrium radial electric field points inward). The theory also predicts the possibility of additional instability, when the $\vec{E} \times \vec{B}$ rotation is present (the rotationally driven trapped - particle mode).

The development of a radial electric field $E_{\rho}$ due to toroidal rotation has been studied for the toroidal geometry ${ }^{10}$. The ion distribution function becomes Maxwellian with temperature uniform on the magnetic surface. The poloidal flow decays. The density is distributed over magnetic flux surfaces by the Boltzmann factor with the effective potential energy that is the sum of a centrifugal potential and an electrostatic potential required for charge neutrality. The usual small gyroradius expansion of the Fokker-Planck equation was carried out, and it was shown, that the effects of centrifugal and Coriolis forces on the particle motion affect the transport.

The rotational equilibrium in cylindrical, non-neutral plasma with an axial magnetic field has been analysed in detail ${ }^{11}$.

The poloidal rotation of plasma due to the $\vec{E} \times \vec{B}$ drift has been studied in tokamaks (the so-called $H$ - mode of improved confinement) with a radial electric field and strong axial magnetic field. The $\vec{E} \times \vec{B}$ drift rotates the plasma poloidally (when the radial electric field exists). In non- $H$-mode plasmas the poloidal rotation frequency is typically much less than the toroidal rotation frequency in tokamaks. The toroidal geometry of tokamaks becomes important in some theories of poloidal spin-up ${ }^{12}$. The poloidal rotation instability (resulting from the poloidally asymmetric diffusion) leads to poloidal velocity shears which may quell the microturbulence. The nonlinear interplay between the poloidal spin-up and turbulence-driven anomalous transport leads to bifurcated equilibria (the $L$ to $H$ transition mode in tokamaks). The tokamaks can spontaneously develop a poloidal velocity shear (the "P-S" flows). The spin-up persists even under conditions where the growth rate is sonic ${ }^{13}$.

The rotation is well known to induce new poles in the perturbed distribution function. The so-called resonant diochotron instability ${ }^{14,15}$ involves the Landau damping at the resonant frequency $\omega=I \Omega(I$ is an integer, $\Omega$ is the rotation frequency). The role of Landau damping in cross-field electron beams and in an inviscid shear flow has been considered for the cylindrical geometry (perturbations with $k_{z}=0$ ) with unperturbed angular velocity $\omega_{0}(r)=\frac{1}{2} n_{0}, 0 \leq r<b$ and $\omega_{0}(r)=\frac{1}{2} n_{0}(b / r)^{2}, b<r \leq a$ ( $n_{0}$ is a constant). A formal analysis of the resonance has been given in the Laplace transforms context ${ }^{14}$. The case of a perfectly conducting wall (at the impedance $Z(\omega)=0$ ) was studied, and a slight shift in the eigenfrequencies from their locations for an induced $Z(\omega) \neq 0$ was found. The Landau damping can overcome the resistive destabilization (if there exist gradients in the electron density) for a strictly decreasing electron density in a cylindrical system, so that only one resistively unstable normal mode can exist - the fundamental one.

The diochotron instability of a thin $\left(\frac{r_{2}-r_{1}}{r_{1}} \ll 1\right)$, tenuous $\left(\omega_{p} \ll \omega_{B e}\right)$, cylin- 
drical layer (a single-component plasma) has been investigated in a strong uniform constant magnetic field using the Vlasov equation ${ }^{15}$. The particle gyroradius was of the order of the mean radius of a layer. The theoretical model does resemble the geometry of some experiments which rely on the high energy particles injection. Two distribution functions were considered, which give the same density $\left(\rho(r) \sim\left[\Theta\left(\vec{r}-\vec{r}_{1}\right)-\Theta\left(\vec{r}-\vec{r}_{2}\right)\right]\right.$, where $\Theta$ is the Heaviside step function), but are different in the velocity space (the velocity spread is zero, or the particles oscillate around the mean radius). The self-electric field of the equilibrium density (which is strictly radial) was included in the treatment (but not the selfmagnetic field). The stability against electrostatic perturbations with frequency around $-l \omega_{B e}$ (where $l$ is the asimuthal wave number of perturbations) and the infinite wavelength in the $z$-direction has been investigated. The perturbed distribution function was found, and the macroscopic sheared flow was shown to be not a relevant criterion for instability by itself.

The gyrokinetic integral equations, used for studying the ion temperature gradient (ITG)- driven mode in a toroidal geometry at low plasma pressure, have been extended to include the equilibrium ion sheared flows ${ }^{16}$. The parallel and perpendicular sheared flows are destabilizing and stabilizing mechanisms, respectively. The gyrokinetic integral code was used to explore the sheared flows' effects on the ITG mode in a sheared slab geometry ${ }^{17}$. The mode structure and eigenfrequencies predicted by the integral code differ from the results derived by the differential approach (for higher radial eigenmodes).

The further development of the above mentioned works and some new ideas are presented in this work. The purposes of the paper are:

1) to show the separation of charges and to obtain rigorous expressions for electric and magnetic self-fields in rotating plasma;

2) to demonstrate the self-sustained rotation origin;

3) to derive the perturbed distribution function and to find the location of poles (the terms in the Landau damping);

4) to propose the local-rotational description of plasma.

The consideration is self-consistent in its character (from the first principles) and leans upon the microscopic properties of a matter, rather than upon the continuous medium (fluid) models. The description can be applied to free rotating plasma objects (astronomical, for example) with some arbitrary shape and composition, to plasma devices with cylindrical symmetry in particular (the arbitrary geometry is included as the limits of integration only).

The peculiarities, which differentiate this work from the cited papers, are as follows:

- Not only the radial electric self-field $E_{\rho}(\rho, z)$, but the magnetic self-field $B_{z}(\rho, z)$ and smaller self-fields $E_{z}(\rho, z)$ and $B_{\rho}(\rho, z)$ are taken into consideration (note that all these components are functions not only of $\rho$ but of $z$ as well). This consideration is necessary, since the behaviour of particles changes qualitatively: the solution depends not only on the small ratio $\frac{v}{c}$, but on arbitrary ratio of character- 
istic velocities as well. All quantities (density, charge density, local composition, etc.) are not externally given but can be evaluated in a self-consistent manner.

- The possibility of a steady-state rotation of plasma is shown. The self-sustained rotation of initially neutral plasma origins in the external magnetic field $\vec{B}_{0}$ as a consequence of the $\vec{E} \times \vec{B}$ drift ( $\vec{E}$ is the self-field).

- The perturbed distribution function is found (rigorously) proceeding from the Vlasov equation. The approach differs in carrying through the extra terms due to $k_{z}$, a self-consistent radial centrifugal force, the self-fields and in allowing the wave amplitudes to be space-dependent.

- The new poles are shown to exist, and the location of these poles is found (the new terms in the Landau damping appear). Since the wave propagation in rotating plasma (an accelerated medium) differs from that in ordinary plasma, the local-rotational description of plasma is supposed to be possible (in the strong turbulence case, for example).

\section{THE ELECTRIC AND MAGNETIC FIELDS IN ROTATING PLASMA}

Rotating plasma possesses some peculiarities as compared to rotating objects which consist of neutral particles. We start with the rigid rotation for a simple explanation only (the substitutions for an arbitrary rotation are given below). The existence of the centrifugal force

$$
\vec{F}=m \Omega^{2} \vec{\rho},
$$

(where $\vec{\Omega}$ is the angular frequency of the system, $m$ is the particle mass, $\vec{\rho}$ is its distance from the axis of rotation) causes different effects on particles of different masses. As a result of these different effects, the $\rho$-dependence of particle concentration is bound to be different for particles of different masses.

According to the Boltzmann distribution,

$$
n_{\alpha}=n_{0 \alpha} \exp \left(\frac{-U_{\alpha}}{k T}\right),
$$

where $T$ is the system temperature, $k$ is the Boltzmann constant, $n_{0 \alpha}$ is the particle concentration on the axis of rotation, $U_{\alpha}$ is the potential energy of $\alpha$ sort particles. In the case of neutral particles

$$
U_{\alpha}=-\frac{m_{\alpha} \Omega^{2} \rho^{2}}{2}
$$

The $n_{\alpha}(\rho)$ dependences for hydrogen plasma are shown schematically in Fig.1. Here $R_{0}$ is the size of a system (the distance from the axis of rotation); symbol $\alpha$ is either $H$ (hydrogen) or $e$ (electron). 


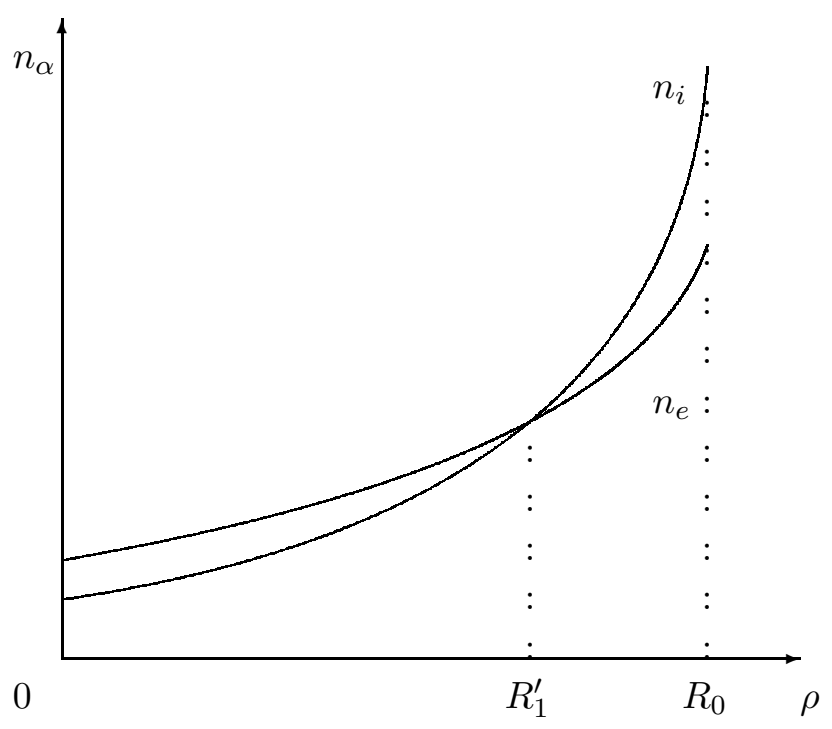

FIG.1. The dependences $n_{\alpha}(\rho)$.

However, plasma consists of charged particles. Since the particles of different masses in plasma have different charges, this partial separation of particles produces a partial separation of charges. The negatively charged region is bound to lie near the axis of rotation, whereas the positively charged region lies near the system boundary $\left(R_{0}\right)$. The electric field $\vec{E}_{0}(\rho, z)$ (in the polar coordinate system: the $\vec{\Omega}$ direction is the z direction) exists as a result of charge separation. This field opposes the considerable separation of charges.

As illustrated in Fig.1, there is some distance from the axis of rotation $R_{1}^{\prime}(z)$, where the local charge equals zero. For $0 \leq \rho<R_{1}^{\prime}(z)$ the plasma is negatively charged on the average; for $R_{1}^{\prime}(z)<\rho \leq R_{0}$ the plasma is positively charged.

The partial separation of charges gives rise to the $\rho$-dependence of charge density; that is, the plasma possessing the given charge density moves round a circle of definite radius. Therefore, circulating currents are inside the rotating plasma despite the fact, that all particles revolve with $\vec{\Omega}$ on the average $(\vec{\Omega}$ has a distinct direction). For $0<\rho<R_{1}^{\prime}$ the current is opposite to the plasma rotation direction (since the charge is negative), whereas for $R_{1}^{\prime}<\rho \leq R_{0}$ the current flows in the direction of rotation (since the charge is positive). In the general case the magnetic actions of currents are not compensated, and the magnetic field $\vec{B}$ exists. This is the magnetic field of a solenoid, whose axis coincides with the axis of system rotation.

For rotating, fully ionized plasma (near macroscopic equilibrium), which consists of elements with atomic number $N_{i}$, it follows in polar coordinates $\rho, \varphi, z(z$ 
axis coincides with the $\vec{\Omega}$ direction), that $n_{e}, n_{i}$ can be taken from (2) with

$$
\begin{gathered}
U_{e}=-\frac{m \Omega^{2} \rho_{1}^{2}}{2}+e \int_{0}^{\rho_{1}} E_{0 \rho}\left(\rho^{\prime}, z_{1}\right) d \rho^{\prime}+e \int_{0}^{z_{1}} E_{0 z}\left(\rho_{1}, z^{\prime}\right) d z^{\prime}+ \\
\int_{0}^{\rho_{1}} \frac{e B_{z}}{c} \Omega \rho^{\prime} d \rho^{\prime}-\int_{0}^{z_{1}} \frac{e B_{\rho}}{c} \Omega \rho_{1} d z^{\prime}, \\
U_{i}=-\frac{M \Omega^{2} \rho_{1}^{2}}{2}-e N_{i} \int_{0}^{\rho_{1}} E_{0 \rho}\left(\rho^{\prime}, z_{1}\right) d \rho^{\prime}-e N_{i} \int_{0}^{z_{1}} E_{0 z}\left(\rho_{1}, z^{\prime}\right) d z^{\prime}- \\
\int_{0}^{\rho_{1}} \frac{e B_{z}}{c} \Omega \rho^{\prime} d \rho^{\prime}+\int_{0}^{z_{1}} \frac{e B_{\rho}}{c} \Omega \rho_{1} d z^{\prime},
\end{gathered}
$$

where $n_{e}(\rho, z)$ is the electron concentration, $n_{i}(\rho, z)$ is the ion concentration, $-e$ is the electron charge $(e>0), m$ is the electron mass, $M$ is the ion mass, $T_{e}=$ $T_{i}=T$ are electron and ion temperatures, $\vec{r}$ is the radius-vector: $\vec{r}=\rho \overrightarrow{e_{\rho}}+z \overrightarrow{e_{z}}$. The quantities $n_{0 \alpha}$ can be found from conditions: $\int_{(V)} n_{\alpha} d v^{\prime}=N_{\alpha} K_{\alpha}$, where $K_{\alpha}$ is the total number of $\alpha$-sort particles, $N_{e}=1$ and $K_{e}=N_{i} K_{i}+\Delta, \Delta$ is the electron surplus (for non-neutral plasma $\Delta \neq 0$ ).

The $\vec{E}_{0}(\rho, z)$ components can be obtained from the system of integral equations:

$$
E_{0 \rho}(\rho, z)=e \int_{(V)} \frac{q\left(\rho_{1}, z_{1}\right)}{R_{1}^{3}} \rho_{1}\left[\rho-\rho_{1} \cos \left(\varphi_{1}-\varphi\right)\right] d \rho_{1} d z_{1} d \varphi_{1},
$$

where

$$
\begin{gathered}
q\left(\rho_{1}, z_{1}\right)=n_{0 i} \exp \left(-\frac{1}{k T} U_{i}\right)-n_{0 e} \exp \left(-\frac{1}{k T} U_{e}\right), \\
R_{1}=\sqrt{\left(z-z_{1}\right)^{2}+\rho^{2}+\rho_{1}^{2}-2 \rho \rho_{1} \cos \left(\varphi_{1}-\varphi\right)} \\
E_{0 z}(\rho, z)=e \int_{(V)} \frac{q\left(\rho_{1}, z_{1}\right)}{R_{1}^{3}}\left(z-z_{1}\right) \rho_{1} d \rho_{1} d z_{1} d \varphi_{1}
\end{gathered}
$$

$V$ is the system volume (its shape is arbitrary).

Radius $R_{1}^{\prime}$ can be found from the equation $n_{e}\left(R_{1}^{\prime}\right)=n_{i}\left(R_{1}^{\prime}\right)$ using (2), (4)-(8).

It follows from the Biot-Savart law, that the magnetic field is

$$
\vec{B}=\frac{1}{c} \int_{(V)} \frac{\left[\overrightarrow{j_{1}} \times \overrightarrow{R_{1}}\right]}{R_{1}^{3}} d v_{1}
$$

where $c$ is the speed of light, $d v_{1}$ is the region with currents, $R_{1}$ is the distance of this region to the point of observation, $\overrightarrow{j_{1}}$ is the current density.

The magnetic field due to rotation can be obtained from the following expressions:

$$
\begin{gathered}
B_{\rho}=\frac{1}{c} \int_{(V)} \frac{j_{\varphi}\left(\rho_{1}, z_{1}\right)}{R_{1}^{3}}\left(z-z_{1}\right) \rho_{1} d \rho_{1} d z_{1} d \varphi_{1} \\
B_{z}=\frac{1}{c} \int_{(V)} \frac{j_{\varphi}\left(\rho_{1}, z_{1}\right)}{R_{1}^{3}} \rho_{1}\left[\rho_{1} \cos \left(\varphi_{1}-\varphi\right)-\rho\right] d \rho_{1} d z_{1} d \varphi_{1}
\end{gathered}
$$


where

$$
j_{\varphi}\left(\rho_{1}, z_{1}\right)=e \Omega \rho_{1} q\left(\rho_{1}, z_{1}\right)
$$

with substitutions $q\left(\rho_{1}, z_{1}\right)$ from $(7), U_{\alpha}$ from (4), (5) and $\vec{E}_{0}\left(\rho^{\prime}, z^{\prime}\right)$ from (6), (8); the magnetic field near the axis of rotation at the system boundary is $B_{z}$ from (10) for $z=L, \rho=0$, where $2 L$ is the system size along the axis of rotation.

The following substitutions need to be done for rotating plasma of complex composition:

$j_{\varphi} \rightarrow \sum_{l} j_{\varphi}^{l}, n_{0 \alpha} \rightarrow n_{0 \alpha}^{l}$; in the case of unsteady rotation

$$
\frac{\Omega^{2} \rho^{2}}{2} \rightarrow \int_{0}^{\rho} \Omega\left(\rho^{\prime}, z\right) \rho^{\prime} d \rho^{\prime}
$$

For astronomical objects the effects can be considerable; in this case the gravitational force plays important role. Note, that in the general case the field $E_{\varphi} \vec{e}_{\varphi}$ exists for free rotating objects with some arbitrary (non-symmetric) shape. In this case all quantities depend on coordinate $\varphi$ as well. The additional terms

$$
e \int_{0}^{\varphi} E_{0 \varphi} \rho_{1} d \varphi_{1}, \quad-e N_{i} \int_{0}^{\varphi} E_{0 \varphi} \rho_{1} d \varphi_{1}
$$

appear in (4) and (5), respectively. The component $E_{0 \varphi}$ is taken from

$$
E_{0 \varphi}(\rho, z, \varphi)=e \int_{(V)} \frac{q\left(\rho_{1}, z_{1}, \varphi_{1}\right)}{R_{1}^{3}} \rho_{1}^{2} \sin \left(\varphi-\varphi_{1}\right) d \rho_{1} d z_{1} d \varphi_{1}
$$

\section{SOME APPROXIMATION OF THE DIELEC- TRIC CONSTANT TENSOR}

Now we obtain information about the electron part of the dielectric constant tensor $\varepsilon_{\alpha \beta}$ (the ion part can be found in a similar manner). The polarization vector $\vec{P}$ is defined as

$$
\frac{\partial \vec{P}}{\partial t}=\vec{j}, \quad \vec{P}=\vec{P}_{0}+\vec{P}^{\prime} \exp [i(\vec{k} \vec{r}-\omega t)] .
$$

It follows that

$$
P_{\alpha}^{\prime}=\frac{\varepsilon_{\alpha \beta}-\delta_{\alpha \beta}}{4 \pi} E_{\beta}^{,},
$$

where $-i \omega \vec{P}^{\prime}=-e K_{e} \vec{v}, \delta_{\alpha \beta}$ is the Kroneker symbol. The expression for $\varepsilon_{\alpha \beta}$ in a rotating system can be derived directly from the equations of electron motion (the field $\vec{E}$ contains the wave field $\vec{E}^{\prime} \sim e^{-i \omega t}$ ) with the force (exact)

$$
\vec{F}_{e}=-e \vec{E}-\frac{e}{c}[\vec{v} \times \vec{B}]+m \Omega^{2} \rho \vec{e}_{\rho}+2 m[\vec{v} \times \vec{\Omega}]-\frac{e}{c} B_{z} \Omega \rho \vec{e}_{\rho}+\frac{e}{c} \Omega \rho B_{\rho} \vec{e}_{z} .
$$

We suppose that the constant field $\vec{B}$ contains all components $\left(B_{0 \rho}, B_{0 \varphi}, B_{0 z}\right)$ and $\vec{v} \sim e^{-i \omega t}$, i.e. space variations of $\vec{E}^{\prime}$ are neglected. Resolving the equation 
$\vec{v}=i \vec{F}_{e} /(m \omega)$ in terms of $v_{\rho}, v_{\varphi}, v_{z}$ and extracting the $\vec{E}^{\prime}$-dependence only (the constant polarization $\vec{P}_{0}$ and the constant part $\varepsilon_{\alpha \beta}^{(0)}$ exist), one obtains

$$
\varepsilon_{\alpha \alpha}=1-\frac{\Omega_{e}^{2}\left[\omega^{2}-\left(\omega_{B e}^{\alpha}\right)^{2}\right]}{\omega^{2}\left[\omega^{2}-\omega_{1}^{2}\right]},
$$

where $\Omega_{e}=\left(4 \pi K_{e} e^{2} / m\right)^{1 / 2}, \omega_{1}=\sqrt{\left(\omega_{B e}^{\rho}\right)^{2}+\left(\omega_{B e}^{\varphi}\right)^{2}+\left(\omega_{B e}^{z}\right)^{2}}$, $\omega_{B e}^{\rho}=e B_{0 \rho} /(m c), \omega_{B e}^{\varphi}=e B_{0 \varphi} /(m c), \omega_{B e}^{z}=e B_{0 z} /(m c)-2 \Omega$,

$$
\varepsilon_{\alpha \beta}=\frac{\Omega_{e}^{2}\left(\omega_{B e}^{\alpha} \omega_{B e}^{\beta}+i \omega \omega_{B e}^{\gamma}\right)}{\omega^{2}\left[\omega^{2}-\omega_{1}^{2}\right]},
$$

here $\alpha, \beta$ and $\gamma$ are taken from cyclic rearrangements in $\rho, \varphi, z ; \varepsilon_{\alpha \beta}=\varepsilon_{\beta \alpha}^{*}$. The approach requires the following conditions:

$v_{T}\left|k_{\alpha}\right| / \omega \ll 1, \quad v_{T}\left|k_{\beta}\right| / \omega_{B e}^{\alpha} \ll 1$, (the smallness of space $\vec{E}^{\prime}$-variations in the localization region of an electron), i.e. $\omega$ must be large enough; and, besides, $\omega$ must not be near the frequency $\omega_{1}$. It follows from the expressions (14), (15), that the dissipation equals zero:

$$
Q=\frac{i \omega}{16 \pi}\left(\varepsilon_{i k}^{*}-\varepsilon_{k i}\right) E_{i} E_{k}^{*}=0
$$

(the tensor $\varepsilon_{\alpha \beta}$ is Hermitian). The $\varepsilon_{\alpha \beta}$ values determine the wave polarization (which is elliptic in the general case) for large $\omega$. The gyration vector $\vec{G}$ can be found from the equation $D_{\alpha}=\varepsilon_{\alpha \beta} E_{\beta}+i[\vec{E} \times \vec{G}]_{\alpha}$, here $i=\sqrt{-1}$. In this approximation the components $G_{\alpha}$ are:

$$
G_{\alpha}=\frac{\Omega_{e}^{2} \omega_{B e}^{\alpha}}{\omega\left[\omega^{2}-\omega_{1}^{2}\right]},
$$

here $\alpha=\rho, \varphi, z$.

We note in the general case, that the space dependences of fields $\vec{E}, \vec{B}$ and the wave amplitude need to be taken into consideration, in principle (to obtain rigorous expressions for $\varepsilon_{\alpha \beta}$ or damping of waves, for example). One may suppose, that, as a consequence of space nonhomogeneity of all characteristics, the rotating plasma system as a whole can be more stable with respect to external disturbances (the system goes from resonance influences). To impart some energy, the spectrum needs to be rather wide-range.

\section{THE LOCATION OF POLES}

Since the rotating plasma is a nonhomogeneous accelerated medium, the wave propagation has some peculiarities (damping, non-rectilinearness). In the general case the resonance conditions possess a relatively complex structure 
$\psi(\rho, \varphi, z, \vec{B}, \vec{E}, \vec{v})=0$. The derivation of the resonance conditions will be demonstrated below in the context of the linear kinetic description for some special cases. Note, that the elementary approach to the problem can be developed from the analysis of the exact force (13): it is necessary to find such particles $(\vec{v}(\rho, \varphi, z, t))$, for which the work of the wave field $A=\int \vec{F} \vec{v} d t$ is positive for some specific wave (in type, direction, polarization). The value $A$ is

$$
A=\int\left\{-e(\vec{E} \vec{v})+m \Omega^{2} \rho v_{\rho}+\frac{e}{c} \Omega \rho\left(B_{\rho} v_{z}-B_{z} v_{\rho}\right)\right\} d t .
$$

If the mean work is positive, the Landau damping mechanism takes place. In the kinetic description we start from the system of self-consistent field equations:

$$
\begin{gathered}
\frac{\partial f_{\alpha}}{\partial t}+\vec{v}_{\alpha} \frac{\partial f_{\alpha}}{\partial \vec{r}}+\vec{F}_{\alpha} \frac{\partial f_{\alpha}}{\partial \vec{p}}=0, \\
\operatorname{rot} \vec{E}=-\frac{1}{c} \frac{\partial \vec{B}}{\partial t}, \quad \operatorname{div} \vec{B}=0, \\
\operatorname{rot} \vec{B}=\frac{1}{c} \frac{\partial \vec{E}}{\partial t}+\frac{4 \pi}{c} \vec{j}, \quad \operatorname{div} \vec{E}=4 \pi q, \\
q=e \int\left(N_{i} f_{i}-f_{e}\right) d^{3} p, \quad \vec{j}=e \int\left(N_{i} f_{i}-f_{e}\right) \vec{v} d^{3} p,
\end{gathered}
$$

and separate the disturbed terms (caused by the wave propagation):

$$
f_{\alpha}=f_{0 \alpha}+\delta f_{\alpha}, \quad \vec{B}=\vec{B}_{0}+\vec{B}^{\prime}, \quad \vec{E}=\vec{E}_{0}+\vec{E}^{\prime},
$$

where $\alpha$ means either $e$ or $i$. It is believed that

$$
\delta f_{\alpha}, \vec{E}^{\prime}, \vec{B}^{\prime} \sim A(\rho, z) \exp i(\vec{k} \vec{r}-\omega t) .
$$

The undisturbed distribution function (in the rotating system) takes the form:

$$
\begin{gathered}
f_{0 e}=\frac{K_{e} D}{(2 \pi m T)^{3 / 2}} \exp \left[-\frac{U_{0}^{\prime}}{T}\right], \\
U_{0}^{\prime}=\frac{m v^{2}}{2}+U_{e},
\end{gathered}
$$

where $K_{e}$ is the total number of electrons in the volume $V, D$ is the normalization constant, $U_{e}$ is taken from (4). The expression (19) is a solution of the kinetic equation (16) with the force (13). $U_{e}$ is the potential part of electron energy (related with the force $\vec{F}$ ). Note that the nonconservative force $e \vec{v} \times \vec{B}$ is not appeared in the potential at all. All terms with $\vec{B}$ include the factor $\Omega$ and exist as a result of the system rotation only. We use the substitutions

$$
\vec{E}_{0}=E_{0 \rho}(\rho, z) \vec{e}_{\rho}+E_{0 z}(\rho, z) \vec{e}_{z}
$$


and $\quad \overrightarrow{k^{\prime}}=\vec{k}+S(\vec{r}) \vec{r}$, where

$$
S(\vec{r})=-\frac{i}{r^{2}}\left(\vec{r} \frac{\partial \ln A(\rho, z)}{\partial \vec{r}}\right) .
$$

Now we make some remarks.

1) The accounting of the amplitude space-dependence is equivalent to including some imaginary part in $\omega: \omega \rightarrow \omega+i \omega^{\prime}$. For, the value $\omega^{\prime}$ is greater than zero in the case of wave damping, and it follows from (18), (21), that the appropriate wave amplitude $A(\rho, z)$ is decreasing; the unstable case $\omega^{\prime}<0$ is analogous to wave amplitude increasing (see $(18),(21))$. The collisional damping can also be included in the term (21). The reverse problem can be formulated: with the $A(\rho, z)$ dependence known (using a probe wave), one should obtain information about collisional processes (the integral of collisions). However, it is not our intention to follow this way.

2) In the general case of unsteady rotation and gravitational force existence the following substitutions need to be done:

$$
E_{0 \rho} \rightarrow E_{0 \rho 1}=E_{0 \rho}-\frac{m_{e}}{e} g_{\rho}(\rho, z), E_{0 z}^{(1)} \rightarrow E_{0 z 1}^{(1)}=E_{0 z}^{(1)}-\frac{m_{e}}{e} g_{z}(\rho, z),
$$

in $(20)$ :

$$
\frac{m \Omega^{2} \rho^{2}}{2 T} \rightarrow \frac{m}{T} \int_{0}^{\rho} \Omega^{2}\left(\rho_{1}, z\right) \rho_{1} d \rho_{1}+\frac{m}{T} \int \vec{g}\left(\rho^{\prime} z^{\prime}\right) d \vec{r}^{\prime}
$$

where

$$
\vec{g}(\rho, z)=G \frac{M(\rho) \vec{r}}{r^{3}}
$$

$G$ is the gravitational constant, $M(\rho)$ is the mass in the $0 \leq \rho^{\prime} \leq \rho$ region; in(13):

$$
m \Omega^{2} \rho \vec{e}_{\rho} \rightarrow m \Omega^{2}(\rho, z) \rho \vec{e}_{\rho}+m \vec{g}(\rho, z) .
$$

The derivation of the disturbed distribution function is demonstrated in Appendix.

Of main interest here are the poles of $\delta f_{e}$ (they contribute to the Landau damping). As a consequence of different regimes of particles behaviour (see Appendix), the resonance conditions differ depending on the particles velocity. For $a<1$ the term $Q(\varphi-\tau)$ does not have any pole in an explicit form (see (A8) in Appendix). The imaginary part of the dielectric constant tensor arises from the imaginary part of $\delta f_{M}$ and from the integration of the exponent ${ }^{18}$. We consider the case $|a| \ll 1$, i.e. $v_{\perp}$ is large enough:

$$
v_{\perp} \gg \frac{\Omega_{1}^{2} \rho}{\omega_{B e}^{(z)}}, \quad v_{\perp} \gg \frac{\omega_{B e}^{(\rho)} v_{z}}{\omega_{B e}^{(z)}} .
$$

All terms (see (A8) in Appendix) can be expanded as a power series in $a$. Integrating over the region $\tau \rightarrow 0$ (which is most considerable for small $|a|$ ), one 
obtains

$\delta f_{e}=Q(\varphi) \int_{0}^{\infty} \exp \left\{-i \tau Y_{1}\right\} d \tau$, where

$$
Y_{1}=\frac{A_{1}}{1+\sin \varphi+\cos \varphi}+A_{1} a \cos \left(\varphi+\varphi_{0}\right)+d_{1}+2 D-\left(d_{1}+D\right) a \sin \left(\varphi+\varphi_{0}\right),
$$

i.e. $\delta f_{e}=-i Q(\varphi) / Y_{1}$. To find the location of poles, it is assumed, that $S=0$; as a result, we have the resonance conditions:

$$
\begin{gathered}
\frac{k_{z} v_{z}-\omega-v_{\perp} k_{\perp} \sin \left(\varphi+\varphi_{0}+\varphi_{3}+\alpha\right)}{\omega_{B e}^{(z)}}= \\
\frac{k_{\perp} v_{\perp}^{2}\left\{\sin \left(\varphi_{3}+\alpha\right)-2 \cos \left(\varphi_{3}+\alpha\right)[1+\cos \varphi+\sin \varphi]\right\}}{(1+\cos \varphi+\sin \varphi) \sqrt{\Omega_{1}^{4} \rho^{2}+v_{z}^{2}\left(\omega_{B e}^{(\rho)}\right)^{2}}} .
\end{gathered}
$$

Thus, the space-dependence of physical quantities leads to considerable change in the resonance conditions, except $k_{\perp}=0$ (for which the well known result follows: $\left.k_{z} v_{z}-\omega=0\right)$. Note, that the resonance conditions depend on the space variable $\rho$ and angle $\alpha$. Besides, the conditions (22) are nonlinear with respect to $v_{\perp}, \varphi, v_{z}$, i.e. in the general case two of these variables cannot be chosen arbitrarily. Therefore, the resonance region decreases.

In spite of the existence of poles in explicit form for $|a|=1$ and $b_{2}=1$, the latter conditions are additional; therefore, the resonances don't contribute to the Landau damping after integration in (A9) (see Appendix).

Now we consider the case $|a|>1$. One can easily see from the structure of term $Q(\varphi-\tau)$, that there are the poles of the integrand at $a>1$ for

$$
\varphi-\tau+\varphi_{0}=\arcsin \left(-\frac{1}{a}\right)
$$

However, for the additional Landau damping the oscillations of the exponent in (A8) (see Appendix) near these poles should be studied. For $a>1$ the oscillations do not increase for $\varphi+\varphi_{0}=-\arcsin (1 / a)$ and $\tau=2 n \pi, n=0,1,2, \ldots$. However, expanding $Y(\varphi, \tau)$ in $\tau$ near $\tau=2 n \pi$ for $n=1,2, \ldots$, one can see that the integration in the limits from $-\infty$ to $+\infty$ in (A8) gives zero. It follows for $n=0$, that $\delta f_{e}=Q(\varphi) \int_{0}^{\infty} \exp \left\{-i \tau Y_{2}\right\} d \tau$, where

$Y_{2}=\frac{A_{1}}{1+\sin \varphi+\cos \varphi}+\frac{A_{1} a \cos \left(\varphi+\varphi_{0}\right)}{1+a \sin \left(\varphi+\varphi_{0}\right)}+D-\frac{\left(d_{1}+D\right) \cos \varphi_{1}}{\sqrt{a^{2}-1}\left(\sin \left(\varphi+\varphi_{0}\right)+\sin \varphi_{1}\right)}$.

Therefore, $\delta f_{e}=-i Q(\varphi) / Y_{2}$. The third term in $Y_{2}$ is negligible near $\varphi+\varphi_{0}=$ $-\arcsin (1 / a)$; the first term is also negligible, except $\varphi=\pi, 3 \pi / 2$. We suppose $S=0$ and, using the definition of $\varphi_{1}$, obtain the resonance conditions:

$$
k_{z} v_{z}+k_{\perp} v_{\perp} \sin \left(\varphi_{3}+\alpha+\varphi_{1}\right)-\omega=0 .
$$

Since $\varphi$ is fixed, the resonance doesn't contribute to the Landau damping. The expression for $Y_{2}$ can be used for finding approximate location of poles in the 
case of $|a| \gg 1$. The latter resonance gives the Landau damping. The resonance conditions are:

$$
k_{z} v_{z}+k_{\perp} v_{\perp} \sin \left(\varphi_{3}+\alpha-\varphi-\varphi_{0}\right)-\omega=-\frac{k_{\perp} v_{\perp} \sin \left(\alpha+\varphi_{3}\right) \sin \left(\varphi+\varphi_{0}\right)}{1+\cos \varphi+\sin \varphi} .
$$

We can see the transformation of the resonance conditions, which is a consequence of plasma motion and inhomogeneity. Note, that (24) gives rise to the well known condition $k_{z} v_{z}-\omega=0$ for $k_{\perp}=0$. Thus, in the general case there exist resonance particles (poles in $\delta f_{e}$ ), which contribute to the Landau damping.

We make some remarks on the local-rotational description of plasma. One can imagine that in a variety of cases the same system is composed of different subsystems. The question of the scale of averaging is not trivial. Since plasma characteristics depend on the regime in it, it is clear that the averaging over different local subsystems can lead to different results. Here the question arises, what particular averaging is correct? It is possible that this case just represents the local-rotational regime (since there is additional damping as waves propagate in an accelerated medium). The basic ideas of the description consist in the following. Instead of using averaged plasma characteristics for finding some physical quantity, we shall seek the quantity in a small rotating subsystem with definite parameters (any plasma particles are involved in the local rotational movements). The net result (the specific physical quantity under investigation in a plasma system as a whole) can be obtained by averaging the quantity over all possible directions of subsystem rotation. Actually, we can imagine that the plasma (even though it is collisionless) consists of rotating parts with radius $r \sim r_{D}$ and linear velocity $v \sim v_{m}$, where $r_{D}$ is the Debye radius

$$
\frac{1}{r_{D}}=\sqrt{\frac{4 \pi e^{2}}{T} \sum_{\alpha} K_{\alpha} N_{\alpha}^{2}},
$$

$v_{m}$ is the most probable velocity

$$
v_{m}=\sqrt{\frac{2 T}{m}} .
$$

Therefore, the characteristic frequency of the local rotation is

$$
\Omega_{l} \sim \frac{v_{m}}{r_{D}}
$$

(collisions produce local rotations with $r \leq \frac{l_{0}}{2}$, where $l_{0}$ is the free path length).

In the general case the solution (but not equations) for a physical quantity under study (transport coefficients, for example) should be averaged over the sequence of local rotating "cells". For a "cell" the kinetic equation takes the form (for electrons):

$$
\frac{\partial f_{e}}{\partial t}+\vec{v} \frac{\partial f_{e}}{\partial \vec{r}}+\left(-e \vec{E}-\frac{e}{c}[\vec{v} \times \vec{B}]+2 m\left[\vec{v} \times \vec{\Omega}_{l}\right]-\right.
$$




$$
\left.\frac{e \Omega_{l} \rho}{c}\left[B_{z} \vec{e}_{\rho}-B_{\rho} \vec{e}_{z}\right]+m \Omega_{l}^{2} \vec{\rho}\right) \frac{\partial f_{e}}{\partial \vec{p}}=\frac{f_{e}-f_{0 e}}{\tau} .
$$

This way of inclusion of local regimes (with subsequent averaging over all directions of $\vec{\Omega}_{l}$ ) can be adaptable to the strong turbulence.

\section{THE SELF-SUSTAINED ROTATION}

First of all, we note that any rotation induces some additional electric field $\vec{E}_{0}$, which is directed towards the axis of rotation (z-axis). Assume that the initially non-rotating plasma system resides in the external magnetic field $\vec{B}_{0}=\vec{e}_{z} B_{0}$. 1) If the electron surplus exists $(\Delta>0)$, then the radial field $\vec{E}_{0 \rho}$ is directed to the $z$-axis. The $\vec{E}_{o \rho} \times \vec{B}$ drift of charged particles results in the movement round a circle. The originated centrifugal force causes partial separation of charges. Therefore, the field $\left|\vec{E}_{0 \rho}\right|$ will increase resulting in sustaining of rotation. Thus, a collective rotation with $\vec{\Omega}$ directed as $\vec{B}$ (parallel) arises spontaneously in a plasma system with an electron surplus.

2) If the ion surplus exists (i.e. $\Delta<0$ ), then the radial field $\vec{E}_{0 \rho}$ is directed from the $z$ axis. The $\vec{E}_{0 \rho} \times \vec{B}$ drift initiates rotation with $\vec{\Omega}$ being antiparallel to $\vec{B}$. However, the partial separation of charges, which is caused by a centrifugal force, results in decreasing $\left|E_{0 \rho}\right|$. This effect counteracts the rotation. Since there exists the rotation with $\vec{\Omega}$ antiparallel to $\vec{B}$ in a positive (only) particle system (it is some limit), there exists some critical value of the ion surplus $\Delta_{c}$ $\left(\Delta=\Delta_{c}<0\right)$, starting from which the spontaneous rotation of plasma $(\vec{\Omega}$ antiparallel to $\vec{B}$ ) is possible.

We shall show that the spontaneous rotation with $\vec{\Omega}$ parallel to $\vec{B}$ can arise in an initially neutral plasma system $(\Delta=0)$. The condition, under which the selfrotation arises, is $\Omega R_{0}<v_{d}$; here $v_{d}$ is the drift velocity, i.e. $B_{0} \Omega R_{0} / c<-E_{0 \rho}$. The equality in the condition is possible for $\Omega=0$ and $\Omega=\Omega_{\text {self }}$. Which equilibrium is stable? We note, that the equiprobable rotational small disturbances $(\vec{\Omega} \rightarrow 0$; or, factually, the ratio of the rotational energy to $k T$ is small) with $\vec{\Omega}$ parallel and antiparallel to $\vec{B}$ are not equivalent for the plasma system as a whole. As a result, plasma will rotate on the average in some definite direction: any rotational disturbance produces the radial field $\vec{E}_{0 \rho}$ directed toward the axis of rotation, but the appeared $\vec{E}_{o \rho} \times \vec{B}$ drift promotes rotational disturbances with $\vec{\Omega}$ parallel to $\vec{B}$ only. We have for the other limit (the case of externally initiated large $\vec{\Omega}$, i.e. $\left.M_{i} \Omega^{2} \rho^{2} /(2 k T) \gg 1\right)$ the inequality $\Omega \rho_{0}>v_{d}$, since the limitations on $E_{0 \rho}$ always exist for any plasma system: $-\infty<E_{0 \rho}^{\min } \leq E_{0 \rho} \leq E_{0 \rho}^{\max }<\infty$. Therefore, the drift opposes the rotation. Thus, the rotation tends to some equilibrium $\Omega_{\text {self }} \neq 0$ for $\Delta=0$.

In the general case, when the rotation $\left(\vec{\Omega}\right.$ is parallel to $\left.\vec{B}_{0}\right)$ arises by any reason, the separation of charges and electric field arise. As a result, the $\vec{E} \times \vec{B}$ drift of charged particles (the movement round a circle) arises. This movement 
will either add to the initial rotation or subtract from it. Eventually, the stable rotation is established. From the equation

$$
v_{d}=\Omega(\rho, z) \rho,
$$

where the drift velocity is

$$
\vec{v}_{d}=-\frac{c E_{0 \rho}(\rho, z) \vec{e}_{\varphi}}{B_{0}}
$$

it follows, that the stable rotation frequency is

$$
\Omega_{\text {self }}(\rho, z)=-\frac{c E_{0 \rho}(\rho, z)}{\rho B_{0}}
$$

with $E_{0 \rho}(\rho, z)$ taken from (6) and $\left|B_{z}\right| \ll\left|B_{0}\right|$.

Under an assumption, that the originated magnetic field $\vec{B}_{z}$ is comparable to $\vec{B}_{0}$, the following substitution needs to be done in equation (25): $B_{0} \rightarrow B_{0}+B_{z}$ with $B_{z}$ taken from (10). In this case the self-sustained rotation of plasma takes place.

We make some remarks.

1) Indeed, to evaluate the rotational state stability one can involve the energy balance. In this case the energy surplus characterizes qualitatively the rate (or time) of transition. However, a rather complex integral system of equations for $\Omega_{\text {self }}$ can be solved numerically (or by a perturbative method) for all specific values of plasma system parameters only.

2) $\Omega_{\text {self }}$ tends to zero for the temperature high enough.

3 ) If the external magnetic field $\vec{B}_{0}$ is zero, then the self-sustained rotation is impossible (the velocity $\rho \Omega_{\text {self }}$ must be greater than the speed of light).

4) If several quasistable field distributions (distinguished by a potential barrier) can exist, then the existence of several quasistable rotating regimes (with $\Omega_{\text {self }}^{j}$ ) is possible.

\section{CONCLUSIONS}

The generation of electric and magnetic nonhomogeneous self-fields in rotating plasma can be explained by various actions of the centrifugal force on dissimilar particles. There are different $\rho$-distributions of these particles and the partial separation of charges. Therefore, the electric field arises (see (6),(8)). The magnetic field (see (9),(10)) exists as a result of rotation of charged regions (the magnetic actions are not compensated). As for relation to the physics topics from Sec.I, there are new peculiarities regarding the self-consistent description. All self-fields $E_{\rho}(\rho, z), E_{z}(\rho, z), B_{\rho}(\rho, z), B_{z}(\rho, z)$ are taken into consideration. The self-sustained rotation (which origins from initially neutral state) exists as a result of the particles drifts in the electric self-field and external magnetic field (see 
(29)). This is a new result. The dielectric constant tensor in rotating plasma differs from that in non-rotating plasma. As the result of plasma inhomogeneity and accelerated motion, the resonance conditions change (see (22),(24)). There are new poles in a disturbed distribution function and, therefore, new terms in the Landau damping. The local-rotational description (over rotating "cells") of plasma can be useful.

\section{APPENDIX: Derivation of the disturbed distribution function}

The problem is to obtain the disturbed electron distribution function. We substitute (17-21) and the force (13) into the kinetic equation (16), correct to the second order $\left(\delta f, \overrightarrow{E^{\prime}}, \overrightarrow{B^{\prime}}\right.$ are small). Then it turns out that

$$
\begin{aligned}
& i\left(\vec{k}^{\prime} \vec{v}-\omega\right) \delta f_{e}+\frac{e f_{0 e}}{T}\left(\vec{v} \vec{E}^{\prime}\right)-\frac{e f_{0 e}}{T c} \Omega \rho\left(B_{\rho}^{\prime} v_{z}-B_{z}^{\prime} v_{\rho}\right)+ \\
& m \Omega_{1}^{2}(\rho, z) \vec{\rho} \frac{\partial \delta f_{e}}{\partial \vec{p}}-\frac{e}{c}\left[\vec{v} \times \vec{B}_{01}\right] \frac{\partial \delta f_{e}}{\partial \vec{p}}-e \vec{E}_{0 z}^{(1)} \frac{\partial \delta f_{e}}{\partial \vec{p}}=0
\end{aligned}
$$

where

$$
\begin{gathered}
\Omega_{1}^{2}(\rho, z)=\Omega^{2}-\frac{e E_{0 \rho}(\rho, z)}{m \rho}-\frac{e B_{0 z}}{m c} \Omega, \quad \vec{B}_{01}=\vec{B}_{0}-\frac{2 m c}{e} \vec{\Omega} \\
\vec{E}_{0 z}^{(1)}=\vec{E}_{0 z}-\frac{\Omega \rho}{c} B_{0 \rho} \vec{e}_{z} .
\end{gathered}
$$

Transferring to the cylindrical coordinate system $\left(v_{z}, v_{\perp}, \varphi\right)$ in the velocity space (Fig. 2), one gets

$$
\begin{gathered}
i\left[k_{z} v_{z}+k_{\perp} v_{\perp} \cos \varphi-\omega+S(\vec{r}) \rho v_{\perp} \cos (\varphi-\alpha)+S(\vec{r}) z v_{z}\right] \delta f_{e}+\frac{e f_{0 e}}{T}\left(\vec{v} \vec{E}^{\prime}\right)- \\
\frac{e f_{0 e} \Omega \rho}{T c}\left(B_{\rho}^{\prime} v_{z}-B_{z}^{\prime} v_{\perp} \cos (\varphi-\alpha)\right)+\left\{\Omega_{1}^{2} \rho \cos (\varphi-\alpha)-\omega_{B e}^{\rho} \sin (\varphi-\alpha) v_{z}\right\} \frac{\partial \delta f_{e}}{\partial v_{\perp}}+ \\
{\left[\omega_{B e}^{(z)}-\frac{\Omega_{1}^{2} \rho}{v_{\perp}} \sin (\varphi-\alpha)-\omega_{B e}^{\rho} \cos (\varphi-\alpha) \frac{v_{z}}{v_{\perp}}\right] \frac{\partial \delta f_{e}}{\partial \varphi}+} \\
{\left[\omega_{B e}^{\rho} \sin (\varphi-\alpha) v_{\perp}-\frac{e E_{0 z 1}^{(1)}}{m}\right] \frac{\partial \delta f_{e}}{\partial v_{z}}=0}
\end{gathered}
$$

where $k_{\perp}$ is the given value, $\sin \alpha=\frac{d}{\rho}$, $d$ is the minimum distance from the center to the ray. Then we obtain the equation

$$
\frac{\partial \delta f_{e}}{\partial \varphi}+i \frac{a_{1} \sin \varphi+b_{1} \cos \varphi+d_{1}}{a_{2} \sin \varphi+b_{2} \cos \varphi+1} \delta f_{e}=Q(\varphi),
$$

where

$$
a_{1}=\frac{S(\vec{r}) v_{\perp} d}{\omega_{B e}^{(z)}}, \quad b_{1}=\frac{k_{\perp} v_{\perp}+S(\vec{r}) \sqrt{\rho^{2}-d^{2}} v_{\perp}}{\omega_{B e}^{(z)}}, d_{1}=\frac{k_{z} v_{z}-\omega+S(\vec{r}) v_{z} z}{\omega_{B e}^{(z)}}
$$




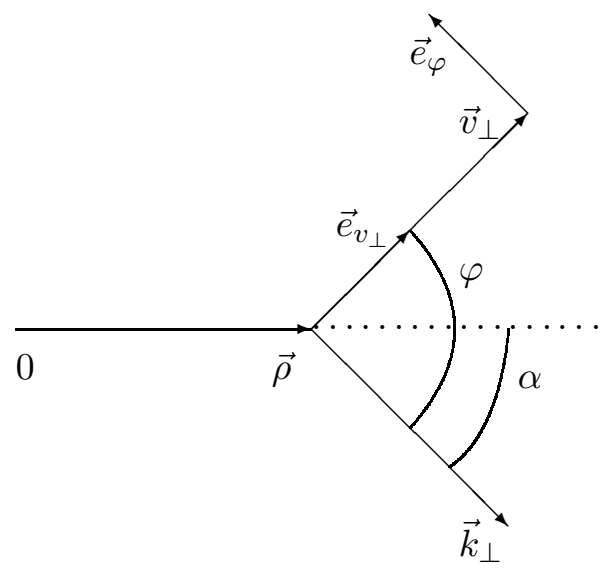

FIG.2. The cylindrical coordinate system in the velocity space.

$$
\begin{gathered}
b_{2}=\frac{\Omega_{1}^{2} \rho}{v_{\perp} \omega_{B e}^{(z)}} \sin \alpha-\frac{\omega_{B e}^{(\rho)} v_{z}}{\omega_{B e}^{(z)} v_{\perp}} \cos \alpha, \quad a_{2}=-\frac{\Omega_{1}^{2} \rho}{v_{\perp} \omega_{B e}^{(z)}} \cos \alpha-\frac{\omega_{B e}^{(\rho)} v_{z}}{\omega_{B e}^{(z)} v_{\perp}} \sin \alpha \\
Q(\varphi)=\frac{W}{\omega_{B e}^{(z)}\left(a_{2} \sin \varphi+b_{2} \cos \varphi+1\right)}, \quad W=-\frac{e f_{0 e}}{T}\left\{\left(\vec{v} \vec{E}^{\prime}\right)-\frac{\Omega \rho}{c}\left[B_{\rho}^{\prime} v_{z}-\right.\right. \\
\left.\left.B_{z}^{\prime} v_{\perp} \cos (\varphi-\alpha)\right]\right\}+\left[\frac{e E_{0 z 1}^{(1)}}{m}-\omega_{B e}^{(\rho)} \sin (\varphi-\alpha) v_{\perp}\right] \frac{\partial \delta f_{e}}{\partial v_{z}}- \\
{\left[\Omega_{1}^{2} \rho \cos (\varphi-\alpha)-\omega_{B e}^{(\rho)} v_{z} \sin (\varphi-\alpha)\right] \frac{\partial \delta f}{\partial v_{\perp}}}
\end{gathered}
$$

The solution of equation (A3) is

$$
\delta f_{e}=e^{-i y(\varphi)} \int_{C}^{\varphi} e^{i y\left(\varphi^{\prime}\right)} Q\left(\varphi^{\prime}\right) d \varphi^{\prime},
$$

where

$$
\frac{d y}{d \varphi}=\frac{a_{1} \sin \varphi+b_{1} \cos \varphi+d_{1}}{a_{2} \sin \varphi+b_{2} \cos \varphi+1}
$$

that is,

$$
\begin{gathered}
y(\varphi)=\frac{b_{1} a_{2}-a_{1} b_{2}}{a_{2}^{2}+b_{2}^{2}} \ln \left|\frac{a_{2} \sin \varphi+b_{2} \cos \varphi+1}{1+\tan \frac{\varphi}{2}}\right|+ \\
\frac{b_{1} b_{2}+a_{1} a_{2}}{a_{2}^{2}+b_{2}^{2}} \varphi+\left(d_{1}-\frac{b_{1} b_{2}+a_{1} a_{2}}{a_{2}^{2}+b_{2}^{2}}\right) \int \frac{d \varphi^{\prime}}{a_{2} \sin \varphi^{\prime}+b_{2} \cos \varphi^{\prime}+1} \\
\int \frac{d \varphi^{\prime}}{a_{2} \sin \varphi^{\prime}+b_{2} \cos \varphi^{\prime}+1}=
\end{gathered}
$$


1)

$$
\begin{gathered}
\frac{2}{\sqrt{1-b_{2}^{2}-a_{2}^{2}}} \arctan \frac{\left(1-b_{2}\right) \tan \frac{\varphi}{2}+a_{2}}{\sqrt{1-b_{2}^{2}-a_{2}^{2}}}, \quad b_{2}^{2}+a_{2}^{2}<1, \\
\text { 2) } \ln \left|\frac{\left(1-b_{2}\right) \tan \frac{\varphi}{2}+a_{2}-\sqrt{b_{2}^{2}+a_{2}^{2}-1}}{\sqrt{b_{2}^{2}+a_{2}^{2}-1}}\right|, \quad b_{2}^{2}+a_{2}^{2}>1, \\
3) \quad \frac{1}{a_{2}} \ln \left|1+a_{2} \tan \frac{\varphi}{2}\right|, \quad b_{2}=1, \\
-\frac{2}{a_{2}+\left(1-b_{2}\right) \tan \frac{\varphi}{2}}, \quad a_{2}^{2}+b_{2}^{2}=1 .
\end{gathered}
$$

Under the $\varphi$-periodicity condition on $\delta f_{e}$ it turns out from (A4) (with the substitution $\varphi^{\prime}=\varphi-\tau$ ), that this expression (note that all functions are $\varphi$-periodic) can possess the $\varphi$-periodicity for $C= \pm \infty$; the value $C=\infty$ obeys all transitions (in limits) to the well-known results only; therefore,

$$
\delta f_{e}=\int_{0}^{\infty} e^{i[y(\varphi-\tau)-y(\varphi)]} Q(\varphi-\tau) d \tau
$$

The substitutions

$$
\begin{gathered}
\varphi_{0}=\arctan \frac{b_{2}}{a_{2}}, \quad A_{1}=-\frac{v_{\perp}\left[k_{\perp} \sin \left(\varphi_{3}+\alpha\right)+\rho S \sin \varphi_{3}\right]}{a \omega_{B e}^{(z)}}, \quad \tan \varphi_{3}=\frac{\Omega_{1}^{2} \rho}{\omega_{B e}^{(\rho)} v_{z}} \\
a^{2}=\frac{\Omega_{1}^{4} \rho^{2}}{v_{\perp}^{2}\left(\omega_{B e}^{(z)}\right)^{2}}+\frac{\left(\omega_{B e}^{(\rho)}\right)^{2} v_{z}^{2}}{\left(\omega_{B e}^{(z)}\right)^{2} v_{\perp}^{2}}, \quad D=\frac{v_{\perp}\left[k_{\perp} \cos \left(\alpha+\varphi_{3}\right)+\rho S \cos \varphi_{3}\right]}{a \omega_{B e}^{(z)}}
\end{gathered}
$$$$
C_{1}=d_{1}+D, \quad \varphi_{1}=\arctan \left(1 / \sqrt{a^{2}-1}\right)
$$

and some rearrangements give:

$$
\begin{gathered}
\delta f_{e}=\int_{0}^{\infty} \exp \{i Y(\varphi, \tau)\} Q(\varphi-\tau) d \tau \\
Y(\varphi, \tau)=A_{1} \ln \left|\frac{\left[1+a \sin \left(\varphi+\varphi_{0}-\tau\right)\right]}{\left[1+a \sin \left(\varphi+\varphi_{0}\right)\right]} \frac{\left[1+\tan \frac{\varphi}{2}\right]}{\left[1+\tan \left(\frac{\varphi-\tau}{2}\right)\right]}\right|-D \tau+C_{1} W_{1}
\end{gathered}
$$

where $W_{1}=$

1) $\quad-\frac{2}{\sqrt{1-a^{2}}} \arctan \left[\frac{\sqrt{1-a^{2}} \sin \frac{\tau}{2}}{\cos \frac{\tau}{2}+a \sin \left(\varphi+\varphi_{0}-\frac{\tau}{2}\right)}\right], \quad|a|<1$,

2) $\frac{1}{\sqrt{a^{2}-1}} \ln \left|\frac{\sin \left(\frac{\varphi+\varphi_{0}+\varphi_{1}-\tau}{2}\right) \cos \left(\frac{\varphi+\varphi_{0}-\varphi_{1}}{2}\right)}{\sin \left(\frac{\varphi+\varphi_{0}+\varphi_{1}}{2}\right) \cos \left(\frac{\varphi+\varphi_{0}-\varphi_{1}-\tau}{2}\right)}\right|, \quad|a|>1$,

$$
\frac{1}{a_{2}} \ln \left|\frac{1+a_{2} \tan \frac{\varphi-\tau}{2}}{1+a_{2} \tan \frac{\varphi}{2}}\right|, \quad b_{2}=1
$$




$$
\begin{gathered}
4) \quad-\frac{2 \sin \frac{\tau}{2}}{\cos \frac{\tau}{2}+\sin \left(\varphi+\varphi_{0}-\frac{\tau}{2}\right)}, \quad|a|=1, \\
Q(\varphi-\tau)=\frac{-1}{1+a \sin \left(\varphi-\tau+\varphi_{0}\right)}\left[\frac { e f _ { 0 e } } { \omega _ { B e } T } \left[\left(\vec{v} \vec{E}^{\prime \prime}\right)-\frac{\Omega \rho}{c}\left(B_{\rho} " v_{z}\right.\right.\right. \\
\left.\left.-B_{z}^{\prime} v_{\perp} \cos (\varphi-\alpha-\tau)\right)\right]+a v_{\perp} \sin \left(\varphi_{3}+\alpha-\varphi+\tau\right) \frac{\partial \delta f_{e}}{\partial v_{\perp}} \\
\left.-\left(\frac{e E_{0 z 1}^{(1)}}{m}-\omega_{B e}^{(\rho)} v_{\perp} \sin (\varphi-\alpha-\tau)\right) \frac{\partial \delta f_{e}}{\partial v_{z}}\right]
\end{gathered}
$$

where the vectors $\vec{E}$ ", $\vec{B}$ " are the vectors $\vec{E}^{\prime}, \vec{B}^{\prime}$ turned at angle $\tau$ in the plane normal to $\vec{B}_{0}$.

Note that expression (A8) is rigorous. As the first approximation, one can use

$$
\frac{\partial \delta f(\varphi-\tau)}{\partial v_{\perp}}=\frac{\partial \delta f_{M}(\varphi-\tau)}{\partial v_{\perp}}, \quad \frac{\partial \delta f(\varphi-\tau)}{\partial v_{z}}=\frac{\partial \delta f_{M}(\varphi-\tau)}{\partial v_{z}},
$$

where the disturbed part of the Maxwell velocity distribution function ${ }^{18}$ is

$$
\begin{gathered}
\delta f_{M}(\varphi)=\int_{0}^{\infty} \exp \left(-i \alpha_{1} \tau^{\prime}-2 i \beta \cos \left(\varphi-\frac{\tau^{\prime}}{2}\right) \sin \frac{\tau^{\prime}}{2}\right) Q^{*}\left(\varphi-\tau^{\prime}\right) d \tau^{\prime}, \\
\alpha_{1}=\frac{k_{z} v_{z}-\omega}{\omega_{B e}^{(z)}}, \quad \beta=\frac{k_{\perp} v_{\perp}}{\omega_{B e}^{(z)}}, \quad Q^{*}(\varphi)=-\frac{e\left(\vec{v} \vec{E}^{\prime}\right) f_{M}}{T \omega_{B e}^{(z)}} .
\end{gathered}
$$

Here $f_{M}$ is the Maxwell velocity distribution function. Note from (A8), that there exist different regimes of particle behaviour (they depend on the velocity of particles). The dielectric constant tensor can be found from

$$
\frac{\varepsilon_{\alpha \beta}-\delta_{\alpha \beta}}{4 \pi} E_{\beta}=\frac{e}{i \omega} \int_{0}^{\infty} \int_{0}^{2 \pi} \int_{-\infty}^{+\infty} v_{\alpha} \delta f_{e} d v_{z} d \varphi v_{\perp} d v_{\perp} .
$$

The dielectric constant tensor differs from that in non-rotating plasma.

\section{References}

[1] H.K. Moffat, Magnetic field generation in electrically conducting fluids (Cambridge University Press, London, New York, 1978).

[2] H. Alfven, Cosmic plasma (D. Reidel Publishing Company, Dordrecht, Holland, 1981).

[3] E.B.Jr. Hooper, Phys. Fluids 13, 96 (1970).

[4] H.M. Stainer, Phys. Fluids 13, 193 (1970).

[5] O. Buneman, J. Electronics and Control 3, 507 (1957). 
[6] V.K. Neil and W. Neckrotte, J. Appl. Phys. 36, 2761 (1965).

[7] R.H. Levy, Phys. Fluids 8, 1288 (1965).

[8] O. Buneman, R.H. Levy and L.M. Linson, J. Appl. Phys. 37, 3203 (1966).

[9] J. Kesner and B. Lane, Phys. Fluids 28, 634 (1985).

[10] F.L. Hinton and S.K. Wong, Phys. Fluids 28, 3082 (1985).

[11] R.C. Davidson, Physics of Non-Neutral Plasmas (Addison-Wesley, 1990).

[12] A.B. Hassam, T.M. Antonsen, Jr.A.M. Dimits, J.F. Drake, P.N. Guzdar, Y.T. Lau, C.S. Liu, K.C. Shaing, P.J. Christenson, W.A. Houlberg and R.D. Hazeltine, Plasma Physics and Controlled Nuclear Fusion Research (International Atomic Energy Agency, Vienna, 1991), vol. 2, p. 311.

[13] A.B. Hassam and J.F. Drake, Phys. Fluids B5, 4022 (1993).

[14] R.J. Briggs, J.D. Daugherty and R.H. Levy, Phys. Fluids 13, 421 (1970).

[15] A. Nocentini, H.L. Berk and R.N. Sudan, J. Plasma Physics 2, 311 (1968).

[16] J.Q. Dong and W. Horton, Phys. Fluids B5, 1581 (1993).

[17] M. Artun, J.V.W. Reynders and W.M. Tang, Phys. Fluids B5, 4072 (1993).

[18] E.M. Lifshitz and L.P. Pitaevskii, Physical Kinetics (Nauka, Moscow, 1973). 